\title{
JOINS, CORONAS AND THEIR VERTEX-EDGE WIENER POLYNOMIALS
}

\author{
MAHDIEH AZARI AND ALI IRANMANESH
}

\begin{abstract}
The vertex-edge Wiener index of a simple connected graph $G$ is defined as the sum of distances between vertices and edges of $G$. The vertex-edge Wiener polynomial of $G$ is a generating function whose first derivative is a $q$-analog of the vertex-edge Wiener index. Two possible distances $D_{1}(u, e \mid G)$ and $D_{2}(u, e \mid G)$ between a vertex $u$ and an edge $e$ of $G$ can be considered and corresponding to them, the first and second vertex-edge Wiener indices of $G$, and the first and second vertex-edge Wiener polynomials of $G$ are introduced. In this paper, we study the behavior of these indices and polynomials under the join and corona product of graphs. Results are applied for some classes of graphs such as suspensions, bottlenecks, and thorny graphs.
\end{abstract}

\section{Introduction}

Throughout the paper, our graphs are considered to be finite, simple, and connected. In theoretical chemistry, the physico-chemical properties of chemical compounds are often modeled by means of molecular-graph-based structure-descriptors, which are also referred to as topological indices $[15,25]$. The vertex version of the Wiener index is the first reported distance-based topological index which was introduced in 1947 by Wiener [26, 27], who used it for modeling the shape of organic molecules and for calculating several of their physicochemical properties. The Wiener index $W(G)$ of a graph $G$ is defined as

$$
W(G)=\sum_{\{u, v\} \subseteq V(G)} d(u, v \mid G),
$$

where $d(u, v \mid G)$ denotes the distance between the vertices $u$ and $v$ of $G$ which is defined as the length of any shortest path in $G$ connecting them. We refer the reader to $[8,10,12,13,23]$ for more information on the Wiener index.

The Wiener polynomial $[17,24]$ of a graph $G$ is defined in terms of a parameter $q$ as

$$
W(G ; q)=\sum_{\{u, v\} \subseteq V(G)} q^{d(u, v \mid G)} .
$$

Received January 8, 2015, accepted July 30, 2015. 2010 Mathematics Subject Classification. 05C12, 05C31, $05 \mathrm{C} 76$.

Key words and phrases. Distance, topological index, graph polynomial, graph operation. Corresponding author: Mahdieh Azari. 
The first derivative of this polynomial at $q=1$ is equal to the Wiener index, i.e., $W^{\prime}(G ; 1)=$ $W(G)$. Details on the Wiener polynomial and its applications can be found in $[9,11,14]$.

The edge versions of the Wiener index based on the distance between edges in a graph $G$ were introduced in 2009 [7, 19, 20]. The first and second edge-Wiener indices of $G$ are denoted by $W_{e_{0}}(G)$ and $W_{e_{4}}(G)$, respectively and defined as

$$
W_{e_{i}}(G)=\sum_{\{e, f\} \subseteq E(G)} d_{i}(e, f \mid G), \quad i \in\{0,4\},
$$

where for distinct pair of edges $e=u v$ and $f=z t$ of $G$, the distances $d_{0}(e, f \mid G)$ and $d_{4}(e, f \mid G)$ are defined as

$$
\begin{gathered}
d_{0}(e, f \mid G)=1+\min \{d(u, z \mid G), d(u, t \mid G), d(v, z \mid G), d(v, t \mid G)\}, \\
d_{4}(e, f \mid G)=\max \{d(u, z \mid G), d(u, t \mid G), d(\nu, z \mid G), d(\nu, t \mid G)\} .
\end{gathered}
$$

It is easy to see that, $d_{0}(e, f \mid G)=d(e, f \mid L(G))$, where $L(G)$ is the line graph of $G$. This implies that, $W_{e_{0}}(G)=W(L(G))$. For details on the edge-Wiener indices see [1, 2, 3, 4, 22] and especially the recent survey [18].

The first and second edge-Wiener polynomials of $G$ are denoted by $W_{e_{0}}(G ; q)$ and $W_{e_{4}}(G ; q)$, respectively and defined in terms of a parameter $q$ as

$$
W_{e_{i}}(G ; q)=\sum_{\{e, f\} \subseteq E(G)} q^{d_{i}(e, f \mid G)}, i \in\{0,4\} .
$$

One can easily see that, $W_{e_{0}}(G ; q)=W(L(G) ; q)$ and the first derivative of the edge-Wiener polynomials at $q=1$ are equal to their corresponding edge-Wiener indices, i.e., $W_{e_{i}}^{\prime}(G ; 1)=$ $W_{e_{i}}(G), i \in\{0,4\}$.

Motivated by definitions of the vertex version and edge versions of the Wiener index, the vertex-edge Wiener indices were defined based on distance between vertices and edges of a graph $[6,20]$. Two possible distances between a vertex $u$ and an edge $e=a b$ of a graph $G$ can be considered. The first distance is denoted by $D_{1}(u, e \mid G)$ and defined as [20],

$$
D_{1}(u, e \mid G)=\min \{d(u, a \mid G), d(u, b \mid G)\},
$$

and the second one is denoted by $D_{2}(u, e \mid G)$ and defined as [6],

$$
D_{2}(u, e \mid G)=\max \{d(u, a \mid G), d(u, b \mid G)\} .
$$

Based on these two distances, two vertex-edge versions of the Wiener index can be defined. The first and second vertex-edge Wiener indices of $G$ are denoted by $W_{\nu e_{1}}(G)$ and $W_{\nu e_{2}}(G)$, respectively and defined as

$$
W_{\nu e_{i}}(G)=\sum_{u \in V(G)} \sum_{e \in E(G)} D_{i}(u, e \mid G), \quad i \in\{1,2\} .
$$


The first and second vertex-edge Wiener polynomials [1] of $G$ are denoted by $W_{v e_{1}}(G ; q)$ and $W_{\nu e_{2}}(G ; q)$, respectively and defined in terms of a parameter $q$ as

$$
W_{v e_{i}}(G ; q)=\sum_{u \in V(G)} \sum_{e \in E(G)} q^{D_{i}(u, e \mid G)}, \quad i \in\{1,2\}
$$

The first derivative of these polynomials at $q=1$ are equal to their corresponding vertex-edge Wiener indices, i.e., $W_{\nu e_{i}}^{\prime}(G ; 1)=W_{\nu e_{i}}(G), i \in\{1,2\}$.

In $[2,5]$, some mathematical properties of these new indices and polynomials were investigated and in [6], the vertex-edge Wiener indices of some chemical graphs were computed. In this paper, we are interested in the type of relationship that exists between the vertex-edge Wiener indices and polynomials of the join and corona product of graphs and their components. Then, we apply our results to compute these indices and polynomials for some classes of graphs by specializing the components in joins and coronas.

\section{Main results}

In this section, we study the behavior of the vertex-edge Wiener polynomials and their related indices under the join and corona product of graphs.

In what follows, for a given graph $G$ we denote by $N_{G}(u)$ the neighborhood of a vertex $u$ in $G$, i.e., the set of all vertices of $G$ adjacent with $u$. The cardinality of $N_{G}(u)$ is called the degree of $u$ in $G$ and is denoted by $\operatorname{deg}_{G}(u)$. One can easily see that,

$$
\sum_{u v \in E(G)}\left|N_{G}(u) \cap N_{G}(v)\right|=3 \Delta(G),
$$

where $\Delta(G)$ is the number of all triangles in $G$.

We denote by $M_{1}(G)$, the first Zagreb index of $G$ which was introduced by Gutman and Trinajstić [16] in 1972, as

$$
M_{1}(G)=\sum_{u \in V(G)} \operatorname{deg}_{G}(u)^{2}=\sum_{u v \in E(G)}\left[\operatorname{deg}_{G}(u)+\operatorname{deg}_{G}(v)\right]
$$

\subsection{Join}

The join $G_{1}+G_{2}+\cdots+G_{n}$ of graphs $G_{1}, G_{2}, \ldots, G_{n}$ with disjoint vertex sets $V\left(G_{1}\right), V\left(G_{2}\right)$, $\ldots, V\left(G_{n}\right)$ and edge sets $E\left(G_{1}\right), E\left(G_{2}\right), \ldots, E\left(G_{n}\right)$ is a graph with the vertex set $V\left(G_{1}\right) \cup V\left(G_{2}\right) \cup$ $\cdots \cup V\left(G_{n}\right)$ and the edge set $E\left(G_{1}\right) \cup E\left(G_{2}\right) \cup \cdots \cup E\left(G_{n}\right) \cup\left\{u v \mid u \in V\left(G_{i}\right), v \in V\left(G_{j}\right), 1 \leq i \neq j \leq n\right\}$. All distinct vertices of $G_{1}+G_{2}+\cdots+G_{n}$ are either at distance 1 or 2 . The vertices at distance 2 are precisely those of $G_{i}$ that are not adjacent in $G_{i}$, for $1 \leq i \leq n$. 
Theorem 2.1. Let $G_{1}, G_{2}, \ldots, G_{n}$ be graphs with $\left|V\left(G_{i}\right)\right|=v_{i}$ and $\left|E\left(G_{i}\right)\right|=e_{i}, 1 \leq i \leq n$, and let $G=G_{1}+G_{2}+\cdots+G_{n}, N=\sum_{i=1}^{n} v_{i}$, and $E=\sum_{i=1}^{n} e_{i}$. Then

$$
\begin{aligned}
W_{v e_{1}}(G ; q)= & \left(N^{2}+2 E-\sum_{i=1}^{n} v_{i}^{2}\right)+\left[\sum_{i=1}^{n} M_{1}\left(G_{i}\right)-3 \sum_{i=1}^{n} \Delta\left(G_{i}\right)+\sum_{i=1}^{n} v_{i}^{3}\right. \\
& \left.-(2 N-1) \sum_{i=1}^{n} v_{i}^{2}-\sum_{i=1}^{n} v_{i} e_{i}+N^{2}(N-1)+E(N-2)\right] q \\
& +\left[3 \sum_{i=1}^{n} \Delta\left(G_{i}\right)-\sum_{i=1}^{n} M_{1}\left(G_{i}\right)+\sum_{i=1}^{n} v_{i} e_{i}\right] q^{2}, \\
W_{\nu e_{2}}(G ; q)= & {\left[3 \sum_{i=1}^{n} \Delta\left(G_{i}\right)+2 \sum_{i=1}^{n} v_{i}^{3}-(3 N+1) \sum_{i=1}^{n} v_{i}^{2}-3 \sum_{i=1}^{n} v_{i} e_{i}\right.} \\
& \left.+N^{2}(N+1)+E(3 N+2)\right] q-\left[3 \sum_{i=1}^{n} \Delta\left(G_{i}\right)+\sum_{i=1}^{n} v_{i}^{3}\right. \\
& \left.-(N+1) \sum_{i=1}^{n} v_{i}^{2}-3 \sum_{i=1}^{n} v_{i} e_{i}+N^{2}+2 E(N+1)\right] q^{2} .
\end{aligned}
$$

Proof. By definition of the join, the polynomial $W_{v e_{r}}(G ; q), r \in\{1,2\}$, can be obtained by adding four polynomials as follows:

$$
\begin{aligned}
W_{v e_{r}}(G ; q) & =\sum_{i=1}^{n} \sum_{u \in V\left(G_{i}\right)} \sum_{e \in E\left(G_{i}\right)} q^{D_{r}(u, e \mid G)}+\sum_{i=1}^{n} \sum_{\substack{j=1 \\
j \neq i}}^{n} \sum_{u \in V\left(G_{i}\right)} \sum_{e \in E\left(G_{j}\right)} q^{D_{r}(u, e \mid G)} \\
& +\sum_{i=1}^{n} \sum_{\substack{j=1 \\
j \neq i}}^{n} \sum_{u, a \in V\left(G_{i}\right)} \sum_{b \in V\left(G_{j}\right)} \sum_{e=a b} q^{D_{r}(u, e \mid G)} \\
& +\sum_{i=1}^{n} \sum_{\substack{j=1 \\
j \neq i}}^{n} \sum_{\substack{k=1 \\
k \neq i, j}}^{n} \sum_{\substack{u \in V\left(G_{i}\right) \\
a \in V\left(G_{j}\right)}} \sum_{b \in V\left(G_{k}\right)} \sum_{e=a b} q^{D_{r}(u, e \mid G)} .
\end{aligned}
$$

Now, we proceed to evaluate each polynomial, separately. So, we consider the following four cases:

Case 1. Let $u \in V\left(G_{i}\right)$ and $e=a b \in E\left(G_{i}\right), 1 \leq i \leq n$. Then

$$
\begin{aligned}
& D_{1}(u, e \mid G)= \begin{cases}0 & u \in\{a, b\}, \\
1 & u \in\left(N_{G_{i}}(a) \cup N_{G_{i}}(b)\right)-\{a, b\}, \\
2 & \text { otherwise, }\end{cases} \\
& D_{2}(u, e \mid G)= \begin{cases}1 & u \in\{a, b\} \cup\left(N_{G_{i}}(a) \cap N_{G_{i}}(b)\right), \\
2 & \text { otherwise. }\end{cases}
\end{aligned}
$$

Hence,

$$
\sum_{i=1}^{n} \sum_{u \in V\left(G_{i}\right)} \sum_{e \in E\left(G_{i}\right)} q^{D_{1}(u, e \mid G)}
$$




$$
\begin{aligned}
& =\sum_{i=1}^{n} \sum_{e=a b \in E\left(G_{i}\right)}\left[\sum_{u \in\{a, b\}} q^{0}+\sum_{u \in\left(N_{G_{i}}(a) \cup N_{G_{i}}(b)\right)-\{a, b\}} q^{1}+\sum_{u \in V\left(G_{i}\right)-\left(N_{G_{i}}(a) \cup N_{G_{i}}(b)\right)} q^{2}\right] \\
& =2 E+\left[\sum_{i=1}^{n} M_{1}\left(G_{i}\right)-3 \sum_{i=1}^{n} \Delta\left(G_{i}\right)-2 E\right] q+\left[\sum_{i=1}^{n} v_{i} e_{i}-\sum_{i=1}^{n} M_{1}\left(G_{i}\right)+3 \sum_{i=1}^{n} \Delta\left(G_{i}\right)\right] q^{2},
\end{aligned}
$$

and

$$
\begin{aligned}
& \sum_{i=1}^{n} \sum_{u \in V\left(G_{i}\right)} \sum_{e \in E\left(G_{i}\right)} q^{D_{2}(u, e \mid G)} \\
= & \sum_{i=1}^{n} \sum_{e=a b \in E\left(G_{i}\right)}\left[\sum_{u \in\{a, b\} \cup\left(N_{G_{i}}(a) \cap N_{G_{i}}(b)\right)} q^{1}+\sum_{u \in V\left(G_{i}\right)-\left[\{a, b\} \cup\left(N_{G_{i}}(a) \cap N_{G_{i}}(b)\right)\right]} q^{2}\right] \\
= & {\left[2 E+3 \sum_{i=1}^{n} \Delta\left(G_{i}\right)\right] q+\left[\sum_{i=1}^{n} v_{i} e_{i}-2 E-3 \sum_{i=1}^{n} \Delta\left(G_{i}\right)\right] q^{2} . }
\end{aligned}
$$

Case 2. Let $u \in V\left(G_{i}\right)$ and $e \in E\left(G_{j}\right)$, where $1 \leq i \neq j \leq n$. Then $D_{1}(u, e \mid G)=D_{2}(u, e \mid G)=1$. So, for $r \in\{1,2\}$,

$$
\begin{aligned}
& \sum_{i=1}^{n} \sum_{\substack{j=1 \\
j \neq i}}^{n} \sum_{u \in V\left(G_{i}\right)} \sum_{e \in E\left(G_{j}\right)} q^{D_{r}(u, e \mid G)}=\sum_{i=1}^{n} \sum_{\substack{j=1 \\
j \neq i}}^{n} \sum_{u \in V\left(G_{i}\right)} \sum_{e \in E\left(G_{j}\right)} q \\
= & q \sum_{i=1}^{n} v_{i} \sum_{\substack{j=1 \\
j \neq i}}^{n} e_{j}=q \sum_{i=1}^{n} v_{i}\left(E-e_{i}\right)=\left(N E-\sum_{i=1}^{n} v_{i} e_{i}\right) q .
\end{aligned}
$$

Case 3. Let $u \in V\left(G_{i}\right)$ and $e=a b$, where $a \in V\left(G_{i}\right), b \in V\left(G_{j}\right), 1 \leq i \neq j \leq n$. Then

$$
D_{1}(u, e \mid G)=\left\{\begin{array}{ll}
0 & u=a, \\
1 & \text { otherwise, }
\end{array} \quad D_{2}(u, e \mid G)= \begin{cases}1 & u \in N_{G_{i}}(a) \cup\{a\}, \\
2 & \text { otherwise. }\end{cases}\right.
$$

Hence,

$$
\begin{aligned}
& \sum_{i=1}^{n} \sum_{\substack{j=1 \\
j \neq i}}^{n} \sum_{u, a \in V\left(G_{i}\right)} \sum_{b \in V\left(G_{j}\right)} \sum_{e=a b} q^{D_{1}(u, e \mid G)} \\
= & \sum_{i=1}^{n} \sum_{\substack{j=1 \\
j \neq i}}^{n} \sum_{a \in V\left(G_{i}\right)} \sum_{b \in V\left(G_{j}\right)}\left[\sum_{u=a} q^{0}+\sum_{u \in V\left(G_{i}\right)-\{a\}} q^{1}\right] \\
= & \sum_{i=1}^{n} v_{i} \sum_{\substack{j=1 \\
j \neq i}}^{n} v_{j}+q \sum_{i=1}^{n} v_{i}\left(v_{i}-1\right) \sum_{\substack{j=1 \\
j \neq i}}^{n} v_{j} \\
= & \sum_{i=1}^{n} v_{i}\left(N-v_{i}\right)+q \sum_{i=1}^{n} v_{i}\left(v_{i}-1\right)\left(N-v_{i}\right) \\
= & N^{2}-\sum_{i=1}^{n} v_{i}^{2}+q \sum_{i=1}^{n}\left[N v_{i}^{2}-v_{i}^{3}-N v_{i}+v_{i}^{2}\right]
\end{aligned}
$$




$$
=N^{2}-\sum_{i=1}^{n} v_{i}^{2}+\left[(N+1) \sum_{i=1}^{n} v_{i}^{2}-\sum_{i=1}^{n} v_{i}^{3}-N^{2}\right] q,
$$

and

$$
\begin{aligned}
& \sum_{i=1}^{n} \sum_{\substack{j=1 \\
j \neq i}}^{n} \sum_{u, a \in V\left(G_{i}\right)} \sum_{b \in V\left(G_{j}\right)} \sum_{e=a b} q^{D_{2}(u, e \mid G)} \\
= & \sum_{i=1}^{n} \sum_{\substack{j=1 \\
j \neq i}}^{n} \sum_{a \in V\left(G_{i}\right)} \sum_{b \in V\left(G_{j}\right)}\left[\sum_{u \in N_{G_{i}}(a) \cup\{a\}} q^{1}+\sum_{u \in V\left(G_{i}\right)-\left(N_{G_{i}}(a) \cup\{a\}\right)} q^{2}\right] \\
= & q \sum_{i=1}^{n}\left(2 e_{i}+v_{i}\right) \sum_{\substack{j=1 \\
j \neq i}}^{n} v_{j}+q^{2} \sum_{i=1}^{n}\left(v_{i}^{2}-2 e_{i}-v_{i}\right) \sum_{\substack{j=1 \\
j \neq i}}^{n} v_{j} \\
= & q \sum_{i=1}^{n}\left(2 e_{i}+v_{i}\right)\left(N-v_{i}\right)+q^{2} \sum_{i=1}^{n}\left(v_{i}^{2}-2 e_{i}-v_{i}\right)\left(N-v_{i}\right) \\
= & {\left[N^{2}+2 N E-2 \sum_{i=1}^{n} v_{i} e_{i}-\sum_{i=1}^{n} v_{i}^{2}\right] q } \\
& +\left[(N+1) \sum_{i=1}^{n} v_{i}^{2}-\sum_{i=1}^{n} v_{i}^{3}+2 \sum_{i=1}^{n} v_{i} e_{i}-N^{2}-2 N E\right] q^{2} .
\end{aligned}
$$

Case 4. Let $u \in V\left(G_{i}\right)$ and $e=a b$, where $a \in V\left(G_{j}\right), b \in V\left(G_{k}\right), 1 \leq i, j, k \leq n, j, k \neq i$, and $j \neq k$. Then $D_{1}(u, e \mid G)=D_{2}(u, e \mid G)=1$. So, for $r \in\{1,2\}$,

$$
\begin{aligned}
& \sum_{i=1}^{n} \sum_{\substack{j=1 \\
j \neq i}}^{n} \sum_{\substack{k=1 \\
k \neq i, j}}^{n} \sum_{u \in V\left(G_{i}\right)} \sum_{a \in V\left(G_{j}\right)} \sum_{b \in V\left(G_{k}\right)} \sum_{e=a b} q^{D_{r}(u, e \mid G)} \\
= & \sum_{i=1}^{n} \sum_{\substack{j=1 \\
j \neq i}}^{n} \sum_{\substack{k=1 \\
k \neq i, j}}^{n} \sum_{u \in V\left(G_{i}\right)} \sum_{a \in V\left(G_{j}\right)} \sum_{b \in V\left(G_{k}\right)} q \\
= & q \sum_{i=1}^{n} v_{i} \sum_{\substack{j=1 \\
j \neq i}}^{n} v_{j} \sum_{\substack{k=1 \\
k \neq i, j}}^{n} v_{k}=q \sum_{i=1}^{n} v_{i} \sum_{\substack{j=1 \\
j \neq i}}^{n} v_{j}\left(N-v_{i}-v_{j}\right) \\
= & q \sum_{i=1}^{n} v_{i}\left[\left(N-v_{i}\right) \sum_{\substack{j=1 \\
j \neq i}}^{n} v_{j}-\sum_{\substack{j=1 \\
j \neq i}}^{n} v_{j}^{2}\right] \\
= & q \sum_{i=1}^{n} v_{i}\left[\left(N-v_{i}\right)^{2}-\left(\sum_{j=1}^{n} v_{j}^{2}-v_{i}^{2}\right)\right] \\
= & q \sum_{i=1}^{n} v_{i}\left[N^{2}-2 N v_{i}+2 v_{i}^{2}-\sum_{j=1}^{n} v_{j}^{2}\right] \\
= & {\left[N^{3}-3 N \sum_{i=1}^{n} v_{i}^{2}+2 \sum_{i=1}^{n} v_{i}^{3}\right] q . }
\end{aligned}
$$


Now, the formulas of the theorem follow by adding the above four polynomials and simplifying the resulting expression.

As a direct consequence of Theorem 2.1, we can compute the vertex-edge Wiener indices of the join of graphs.

Corollary 2.2. Let $G_{1}, G_{2}, \ldots, G_{n}$ be graphs with $\left|V\left(G_{i}\right)\right|=v_{i}$ and $\left|E\left(G_{i}\right)\right|=e_{i}, 1 \leq i \leq n$, and let $G=G_{1}+G_{2}+\cdots+G_{n}, N=\sum_{i=1}^{n} v_{i}$, and $E=\sum_{i=1}^{n} e_{i}$. Then

$$
\begin{aligned}
W_{v e_{1}}(G)= & 3 \sum_{i=1}^{n} \Delta\left(G_{i}\right)-\sum_{i=1}^{n} M_{1}\left(G_{i}\right)+\sum_{i=1}^{n} v_{i}^{3}-(2 N-1) \sum_{i=1}^{n} v_{i}^{2}+\sum_{i=1}^{n} v_{i} e_{i} \\
& +N^{2}(N-1)+E(N-2), \\
W_{v e_{2}}(G)= & 3 \sum_{i=1}^{n} v_{i} e_{i}-3 \sum_{i=1}^{n} \Delta\left(G_{i}\right)-(N-1) \sum_{i=1}^{n} v_{i}^{2}+N^{2}(N-1)-E(N+2) .
\end{aligned}
$$

Using Theorem 2.1 and Corollary 2.2, the formulas for vertex-edge Wiener polynomials and indices of the join of two graphs are easily computed.

Corollary 2.3. Let $G_{1}$ and $G_{2}$ be graphs with $\left|V\left(G_{i}\right)\right|=v_{i}$ and $\left|E\left(G_{i}\right)\right|=e_{i}, 1 \leq i \leq 2$. Then

$$
\begin{aligned}
W_{v e_{1}}\left(G_{1}+G_{2} ; q\right)= & 2\left(e_{1}+e_{2}+v_{1} v_{2}\right)+\left[M_{1}\left(G_{1}\right)+M_{1}\left(G_{2}\right)-3\left(\Delta\left(G_{1}\right)+\Delta\left(G_{2}\right)\right)\right. \\
& \left.+v_{1} v_{2}\left(v_{1}+v_{2}-2\right)+e_{1}\left(v_{2}-2\right)+e_{2}\left(v_{1}-2\right)\right] q \\
& +\left[3\left(\Delta\left(G_{1}\right)+\Delta\left(G_{2}\right)\right)-\left(M_{1}\left(G_{1}\right)+M_{1}\left(G_{2}\right)\right)+v_{1} e_{1}+v_{2} e_{2}\right] q^{2}, \\
W_{v e_{2}}\left(G_{1}+G_{2}, q\right)= & {\left[3\left(\Delta\left(G_{1}\right)+\Delta\left(G_{2}\right)\right)+2\left(e_{1}+e_{2}+v_{1} v_{2}\right)+3\left(e_{1} v_{2}+v_{1} e_{2}\right)\right] q } \\
& -\left[3\left(\Delta\left(G_{1}\right)+\Delta\left(G_{2}\right)\right)-\left(v_{1}+v_{2}-2\right)\left(v_{1} v_{2}+e_{1}+e_{2}\right)\right. \\
& \left.+3\left(e_{1} v_{2}+v_{1} e_{2}\right)\right] q^{2} .
\end{aligned}
$$

Corollary 2.4. Let $G_{1}$ and $G_{2}$ be graphs with $\left|V\left(G_{i}\right)\right|=v_{i}$ and $\left|E\left(G_{i}\right)\right|=e_{i}, 1 \leq i \leq 2$. Then

$$
\begin{aligned}
W_{\nu e_{1}}\left(G_{1}+G_{2}\right)= & 3\left(\Delta\left(G_{1}\right)+\Delta\left(G_{2}\right)\right)-\left(M_{1}\left(G_{1}\right)+M_{1}\left(G_{2}\right)\right)+v_{1} e_{1}+v_{2} e_{2} \\
& +\left(v_{1}+v_{2}-2\right)\left(v_{1} v_{2}+e_{1}+e_{2}\right), \\
W_{\nu e_{2}}\left(G_{1}+G_{2}\right)= & 2 v_{1} v_{2}\left(v_{1}+v_{2}-1\right)+e_{1}\left(2 v_{1}-v_{2}-2\right)+e_{2}\left(2 v_{2}-v_{1}-2\right) \\
& -3\left(\Delta\left(G_{1}\right)+\Delta\left(G_{2}\right)\right) .
\end{aligned}
$$

It is interesting to note that, the formulas of Theorem 2.1 and Corollaries 2.2, 2.3, and 2.4 do not depend on the connectivity of the primary graphs. This allows us to compute the vertex-edge Wiener polynomials and indices of the join of graphs that are not themselves connected. 


\subsection{Corona}

The corona product $G \circ H$ of graphs $G$ and $H$ is a graph obtained by taking one copy of $G$ and $|V(G)|=n$ copies of $H$, and joining the $i$-th vertex of $G$ to every vertex in $i$-th copy of $H$ for $i=1,2, \ldots, n$. The $i$-th copy of $H$ and $i$-th element of $G$ will be denoted by $H_{i}$ and $w_{i}, 1 \leq$ $i \leq n$, respectively. So, we can consider the vertex set of the graph $G$ as $V(G)=\left\{w_{1}, w_{2}, \ldots, w_{n}\right\}$ and the vertex and edge sets of the graph $G \circ H$ as

$$
\begin{aligned}
& V(G \circ H)=V(G) \cup V\left(H_{1}\right) \cup \cdots \cup V\left(H_{n}\right), \\
& E(G \circ H)=E(G) \cup E\left(H_{1}\right) \cup \cdots \cup E\left(H_{n}\right) \cup\left\{w_{i} v \mid v \in V\left(H_{i}\right), 1 \leq i \leq n\right\} .
\end{aligned}
$$

Also, the distance between two distinct vertices $u, v \in V(G \circ H)$ is given by

$$
d(u, v \mid G \circ H)= \begin{cases}d(u, v \mid G) & u, v \in V(G), \\ d\left(u, w_{i} \mid G\right)+1 & u \in V(G), v \in V\left(H_{i}\right), \\ 1 & u v \in E\left(H_{i}\right), \\ 2 & u, v \in V\left(H_{i}\right), u v \notin E\left(H_{i}\right), \\ d\left(w_{i}, w_{j} \mid G\right)+2 & u \in V\left(H_{i}\right), v \in V\left(H_{j}\right), i \neq j .\end{cases}
$$

Theorem 2.5. Let $G$ and $H$ be graphs with $|V(G)|=n,|V(H)|=n^{\prime},|E(G)|=e$, and $|E(H)|=e^{\prime}$. Then

$$
\begin{aligned}
W_{v e_{1}}(G \circ H ; q)= & \left(n^{\prime} q+1\right) W_{v e_{1}}(G ; q)+2\left(n^{\prime} q+1\right)\left(e^{\prime} q+n^{\prime}\right) W(G ; q)+n q^{2}[3 \Delta(H) \\
& \left.-M_{1}(H)+n^{\prime} e^{\prime}\right]+n q\left[M_{1}(H)-3 \Delta(H)+n^{\prime}\left(n^{\prime}-1\right)-e^{\prime}\right]+2 n\left(n^{\prime}+e^{\prime}\right), \\
W_{v e_{2}}(G \circ H ; q)= & \left(n^{\prime} q+1\right) W_{v e_{2}}(G ; q)+2 q\left(n^{\prime} q+1\right)\left(n^{\prime}+e^{\prime}\right) W(G ; q)+n q^{2}\left[n^{\prime}\left(n^{\prime}-1\right)\right. \\
& \left.+e^{\prime}\left(n^{\prime}-4\right)-3 \Delta(H)\right]+n q\left[2 n^{\prime}+5 e^{\prime}+3 \Delta(H)\right] .
\end{aligned}
$$

Proof. By definition of the corona product, the polynomial $W_{v e_{r}}(G \circ H ; q), r \in\{1,2\}$, can be obtained by adding eight polynomials as follows:

$$
\begin{aligned}
& W_{v e_{r}}(G \circ H ; q)=\sum_{u \in V(G)} \sum_{e \in E(G)} q^{D_{r}(u, e \mid G \circ H)}+\sum_{i=1}^{n} \sum_{u \in V(G)} \sum_{e \in E\left(H_{i}\right)} q^{D_{r}(u, e \mid G \circ H)} \\
& +\sum_{i=1}^{n} \sum_{u \in V(G)} \sum_{v \in V\left(H_{i}\right)} \sum_{e=w_{i} v} q^{D_{r}(u, e \mid G \circ H)}+\sum_{i=1}^{n} \sum_{u \in V\left(H_{i}\right)} \sum_{e \in E(G)} q^{D_{r}(u, e \mid G \circ H)} \\
& +\sum_{i=1}^{n} \sum_{u \in V\left(H_{i}\right)} \sum_{e \in E\left(H_{i}\right)} q^{D_{r}(u, e \mid G \circ H)}+\sum_{i=1}^{n} \sum_{\substack{j=1 \\
j \neq i}}^{n} \sum_{u \in V\left(H_{i}\right)} \sum_{e \in E\left(H_{j}\right)} q^{D_{r}(u, e \mid G \circ H)} \\
& +\sum_{i=1}^{n} \sum_{u, v \in V\left(H_{i}\right)} \sum_{e=w_{i} v} q^{D_{r}(u, e \mid G \circ H)}+\sum_{i=1}^{n} \sum_{\substack{j=1 \\
j \neq i}}^{n} \sum_{u \in V\left(H_{i}\right)} \sum_{v \in V\left(H_{j}\right)} \sum_{e=w_{i} v} q^{D_{r}(u, e \mid G \circ H)} .
\end{aligned}
$$


We proceed to evaluate each polynomial, separately. So, we consider the following eight cases:

Case 1. Let $u \in V(G)$ and $e \in E(G)$. Then

$$
D_{r}(u, e \mid G \circ H)=D_{r}(u, e \mid G), \quad r \in\{1,2\} .
$$

So, for $r \in\{1,2\}$,

$$
\sum_{u \in V(G)} \sum_{e \in E(G)} q^{D_{r}(u, e \mid G \circ H)}=\sum_{u \in V(G)} \sum_{e \in E(G)} q^{D_{r}(u, e \mid G)}=W_{\nu e_{r}}(G ; q) .
$$

Case 2. Let $u \in V(G)$ and $e \in E\left(H_{i}\right), 1 \leq i \leq n$. Then

$$
D_{r}(u, e \mid G \circ H)=d\left(u, w_{i} \mid G\right)+1, \quad r \in\{1,2\} .
$$

So, for $r \in\{1,2\}$,

$$
\sum_{i=1}^{n} \sum_{u \in V(G)} \sum_{e \in E\left(H_{i}\right)} q^{D_{r}(u, e \mid G \circ H)}=\sum_{i=1}^{n} \sum_{u \in V(G)} \sum_{e \in E\left(H_{i}\right)} q^{d\left(u, w_{i} \mid G\right)+1}=e^{\prime} q[n+2 W(G ; q)] .
$$

Case 3. Let $u \in V(G)$ and $e=w_{i} v$, where $v \in V\left(H_{i}\right), 1 \leq i \leq n$. Then

$$
D_{1}(u, e \mid G \circ H)=d\left(u, w_{i} \mid G\right), D_{2}(u, e \mid G \circ H)=d\left(u, w_{i} \mid G\right)+1 .
$$

Hence,

$$
\sum_{i=1}^{n} \sum_{u \in V(G)} \sum_{v \in V\left(H_{i}\right)} \sum_{e=w_{i} \nu} q^{D_{1}(u, e \mid G \circ H)}=\sum_{i=1}^{n} \sum_{u \in V(G)} \sum_{v \in V\left(H_{i}\right)} q^{d\left(u, w_{i} \mid G\right)}=n^{\prime}[n+2 W(G ; q)],
$$

and

$$
\sum_{i=1}^{n} \sum_{u \in V(G)} \sum_{\nu \in V\left(H_{i}\right)} \sum_{e=w_{i} \nu} q^{D_{2}(u, e \mid G \circ H)}=\sum_{i=1}^{n} \sum_{u \in V(G)} \sum_{\nu \in V\left(H_{i}\right)} q^{d\left(u, w_{i} \mid G\right)+1}=n^{\prime} q[n+2 W(G ; q)] .
$$

Case 4. Let $u \in V\left(H_{i}\right)$ and $e \in E(G)$, where $1 \leq i \leq n$. Then

$$
D_{r}(u, e \mid G \circ H)=D_{r}\left(w_{i}, e \mid G\right)+1, \quad r \in\{1,2\} .
$$

So, for $r \in\{1,2\}$,

$$
\sum_{i=1}^{n} \sum_{u \in V\left(H_{i}\right)} \sum_{e \in E(G)} q^{D_{r}(u, e \mid G \circ H)}=\sum_{i=1}^{n} \sum_{u \in V\left(H_{i}\right)} \sum_{e \in E(G)} q^{D_{r}\left(w_{i}, e \mid G\right)+1}=n^{\prime} q W_{v e_{r}}(G, q) .
$$

Case 5. Let $u \in V\left(H_{i}\right)$ and $e \in E\left(H_{i}\right), 1 \leq i \leq n$. Then

$$
D_{1}(u, e \mid G \circ H)= \begin{cases}0 & u \in\{a, b\}, \\ 1 & u \in\left(N_{H_{i}}(a) \cup N_{H_{i}}(b)\right)-\{a, b\}, \\ 2 & \text { otherwise, }\end{cases}
$$




$$
D_{2}(u, e \mid G \circ H)= \begin{cases}1 & u \in\{a, b\} \cup\left(N_{H_{i}}(a) \cap N_{H_{i}}(b)\right), \\ 2 & \text { otherwise }\end{cases}
$$

Hence,

$$
\begin{aligned}
\sum_{i=1}^{n} & \sum_{u \in V\left(H_{i}\right)} \sum_{e \in E\left(H_{i}\right)} q^{D_{1}(u, e \mid G \circ H)} \\
\quad= & \sum_{i=1}^{n} \sum_{a b \in E\left(H_{i}\right)}\left[\sum_{u \in\{a, b\}} q^{0}+\sum_{u \in\left(N_{H_{i}}(a) \cup N_{H_{i}}(b)\right)-\{a, b\}} q^{1}+\sum_{u \in V\left(H_{i}\right)-\left(N_{H_{i}}(a) \cup N_{H_{i}}(b)\right)} q^{2}\right] \\
& =2 n e^{\prime}+n q\left[M_{1}(H)-3 \Delta(H)-2 e^{\prime}\right]+n q^{2}\left[n^{\prime} e^{\prime}-M_{1}(H)+3 \Delta(H)\right],
\end{aligned}
$$

and

$$
\begin{aligned}
\sum_{i=1}^{n} \sum_{u \in V\left(H_{i}\right)} \sum_{e \in E\left(H_{i}\right)} q^{D_{2}(u, e \mid G \circ H)} \\
\quad=\sum_{i=1}^{n} \sum_{a b \in E\left(H_{i}\right)}\left[\sum_{u \in\{a, b\} \cup\left(N_{H_{i}}(a) \cap N_{H_{i}}(b)\right)} q^{1}+\sum_{u \in V\left(H_{i}\right)-\left[\{a, b\} \cup\left(N_{H_{i}}(a) \cap N_{H_{i}}(b)\right)\right]} q^{2}\right] \\
=n q\left[2 e^{\prime}+3 \Delta(H)\right]+n q^{2}\left[n^{\prime} e^{\prime}-2 e^{\prime}-3 \Delta(H)\right] .
\end{aligned}
$$

Case 6. Let $u \in V\left(H_{i}\right)$ and $e \in E\left(H_{j}\right)$, where $1 \leq i \neq j \leq n$. Then

$$
D_{r}(u, e \mid G \circ H)=d\left(w_{i}, w_{j} \mid G\right)+2, \quad r \in\{1,2\} .
$$

So, for $r \in\{1,2\}$,

$$
\begin{aligned}
\sum_{i=1}^{n} \sum_{\substack{j=1 \\
j \neq i}}^{n} \sum_{u \in V\left(H_{i}\right)} \sum_{e \in E\left(H_{j}\right)} q^{D_{r}(u, e \mid G \circ H)} & =\sum_{i=1}^{n} \sum_{\substack{j=1 \\
j \neq i}}^{n} \sum_{u \in V\left(H_{i}\right)} \sum_{e \in E\left(H_{j}\right)} q^{d\left(w_{i}, w_{j} \mid G\right)+2} \\
& =2 n^{\prime} e^{\prime} q^{2} W(G ; q) .
\end{aligned}
$$

Case 7. Let $u, v \in V\left(H_{i}\right)$ and $e=w_{i} v, 1 \leq i \leq n$. Then

$$
D_{1}(u, e \mid G \circ H)=\left\{\begin{array}{ll}
0 & v=u, \\
1 & \text { otherwise, }
\end{array} \quad D_{2}(u, e \mid G \circ H)= \begin{cases}1 & v \in N_{H_{i}}(u) \cup\{u\}, \\
2 & \text { otherwise. }\end{cases}\right.
$$

Hence,

$$
\begin{aligned}
\sum_{i=1}^{n} \sum_{u, v \in V\left(H_{i}\right)} \sum_{e=w_{i} v} q^{D_{1}\left(u, e \mid G_{\circ} H\right)} & =\sum_{i=1}^{n} \sum_{u \in V\left(H_{i}\right)}\left[\sum_{v=u} q^{0}+\sum_{v \in V\left(H_{i}\right)-\{u\}} q^{1}\right] \\
& =n n^{\prime}+n n^{\prime}\left(n^{\prime}-1\right) q
\end{aligned}
$$

and

$$
\begin{aligned}
\sum_{i=1}^{n} \sum_{u, v \in V\left(H_{i}\right)} \sum_{e=w_{i} \nu} q^{D_{2}(u, e \mid G \circ H)} & =\sum_{i=1}^{n} \sum_{u \in V\left(H_{i}\right)}\left[\sum_{v \in N_{H_{i}}(u) \cup\{u\}} q+\sum_{v \in V\left(H_{i}\right)-\left(N_{H_{i}}(u) \cup\{u\}\right)} q^{2}\right] \\
& =n\left(n^{\prime}+2 e^{\prime}\right) q+n q^{2}\left[n^{\prime}\left(n^{\prime}-1\right)-2 e^{\prime}\right] .
\end{aligned}
$$


Case 8. Let $u \in V\left(H_{i}\right)$ and $e=w_{j} v$, where $v \in V\left(H_{j}\right), 1 \leq i \neq j \leq n$. Then

$$
D_{1}(u, e \mid G \circ H)=d\left(w_{i}, w_{j} \mid G\right)+1, D_{2}(u, e \mid G \circ H)=d\left(w_{i}, w_{j} \mid G\right)+2 .
$$

Hence,

$$
\begin{aligned}
& \sum_{i=1}^{n} \sum_{\substack{j=1 \\
j \neq i}}^{n} \sum_{u \in V\left(H_{i}\right)} \sum_{v \in V\left(H_{j}\right)} \sum_{e=w_{i} v} q^{D_{1}(u, e \mid G \circ H)} \\
& \quad=\sum_{\substack{i=1 \\
j}}^{n} \sum_{\substack{j=1 \\
j \neq i}}^{n} \sum_{u \in V\left(H_{i}\right)} \sum_{v \in V\left(H_{j}\right)} q^{d\left(w_{i}, w_{j} \mid G\right)+1}=2 n^{\prime 2} q W(G ; q),
\end{aligned}
$$

and

$$
\begin{aligned}
& \sum_{i=1}^{n} \sum_{\substack{j=1 \\
j \neq i}}^{n} \sum_{u \in V\left(H_{i}\right)} \sum_{\nu \in V\left(H_{j}\right)} \sum_{e=w_{i} \nu} q^{D_{2}(u, e \mid G \circ H)} \\
& \quad=\sum_{i=1}^{n} \sum_{\substack{j=1 \\
j \neq i}}^{n} \sum_{u \in V\left(H_{i}\right)} \sum_{v \in V\left(H_{j}\right)} q^{d\left(w_{i}, w_{j} \mid G\right)+2}=2 n^{\prime 2} q^{2} W(G ; q) .
\end{aligned}
$$

Now, the formulas of the theorem follow by adding the above eight polynomials and simplifying the resulting expression.

As a direct consequence of Theorem 2.5, we obtain the following corollary.

Corollary 2.6. Let $G$ and $H$ be graphs with $|V(G)|=n,|V(H)|=n^{\prime},|E(G)|=e$, and $|E(H)|=e^{\prime}$. Then

$$
\begin{aligned}
W_{v e_{1}}(G \circ H)= & \left(n^{\prime}+1\right) W_{v e_{1}}(G)+2\left(n^{\prime}+1\right)\left(n^{\prime}+e^{\prime}\right) W(G) \\
& +n\left[3 \Delta(H)-M_{1}(H)+n e^{\prime}+e n^{\prime}+\left(n^{\prime}+2 e^{\prime}\right)\left(n n^{\prime}-1\right)\right], \\
W_{v e_{2}}(G \circ H)= & \left(n^{\prime}+1\right) W_{v e_{2}}(G)+2\left(n^{\prime}+1\right)\left(n^{\prime}+e^{\prime}\right) W(G) \\
& +n n^{\prime}\left[2 n\left(n^{\prime}+e^{\prime}\right)+n+e-1\right]+n e^{\prime}(n-4)-3 n \Delta(H) .
\end{aligned}
$$

Again, it is interesting to note that, the formulas of Theorem 2.5 and Corollary 2.6 do not include any invariants of $H$ that depend on its connectivity. So, it is possible to apply Theorem 2.5 and Corollary 2.6 to the corona products $G \circ H$ with disconnected $H$. Such cases arise in transitions from kenographs to plerographs, where $H$ is given as an empty graph, i.e., as $\bar{K}_{n}$ for some positive integer $n$.

\section{Examples}

In this section, we present explicit formulas for computing the vertex-edge Wiener polynomials and their related indices for some classes of graphs by specializing components in joins and coronas. 
For a given graph $G$, the graph $K_{1}+G=K_{1} \circ G$ is called the suspension [28] of $G$, where $K_{1}$ denotes a single vertex graph. Using Corollary 2.3 and Corollary 2.4, we get the following results.

Corollary 3.1. Let $G$ be a graph of order $n$ and size e. Then

$$
\begin{aligned}
W_{v e_{1}}\left(K_{1}+G ; q\right) & =2(n+e)+\left[n(n-1)-e+M_{1}(G)-3 \Delta(G)\right] q+\left[n e-M_{1}(G)+3 \Delta(G)\right] q^{2}, \\
W_{\nu e_{2}}\left(K_{1}+G ; q\right) & =[2 n+5 e+3 \Delta(G)] q+[(n-1)(n+e)-3 e-3 \Delta(G)] q^{2}, \\
W_{\nu e_{1}}\left(K_{1}+G\right) & =n e+(n-1)(n+e)-M_{1}(G)+3 \Delta(G), \\
W_{v e_{2}}\left(K_{1}+G\right) & =2 n^{2}+(2 n-3) e-3 \Delta(G) .
\end{aligned}
$$

Star graph $S_{n+1}$ on $n+1$ vertices is the suspension of empty graph on $n$ vertices $\bar{K}_{n}$. Fan graph $F_{n+1}$ and wheel graph $W_{n+1}$ on $n+1$ vertices are also the suspension of $n$-vertex path $P_{n}$ and $n$-vertex cycle $C_{n}$, respectively. So, by the above formulas, the vertex-edge Wiener polynomials and indices of these graphs are obtained, at once.

Corollary 3.2. For $n \geq 3$,

$$
\begin{aligned}
W_{v e_{1}}\left(S_{n+1} ; q\right) & =W_{v e_{1}}\left(K_{1}+\bar{K}_{n}, q\right)=2 n+n(n-1) q, \\
W_{v e_{2}}\left(S_{n+1} ; q\right) & =W_{v e_{2}}\left(K_{1}+\bar{K}_{n}, q\right)=2 n q+n(n-1) q^{2}, \\
W_{v e_{1}}\left(S_{n+1}\right) & =W_{v e_{1}}\left(K_{1}+\bar{K}_{n}\right)=n(n-1), \\
W_{v e_{2}}\left(S_{n+1}\right) & =W_{v e_{2}}\left(K_{1}+\bar{K}_{n}\right)=2 n^{2} .
\end{aligned}
$$

Corollary 3.3. For $n \geq 2$,

$$
\begin{aligned}
W_{v e_{1}}\left(F_{n+1} ; q\right) & =W_{v e_{1}}\left(K_{1}+P_{n}, q\right)=2(2 n-1)+\left(n^{2}+2 n-5\right) q+\left(n^{2}-5 n+6\right) q^{2}, \\
W_{v e_{2}}\left(F_{n+1} ; q\right) & =W_{v e_{2}}\left(K_{1}+P_{n}, q\right)=(7 n-5) q+2\left(n^{2}-3 n+2\right) q^{2}, \\
W_{v e_{1}}\left(F_{n+1}\right) & =W_{v e_{1}}\left(K_{1}+P_{n}\right)=3 n^{2}-8 n+7, \\
W_{v e_{2}}\left(F_{n+1}\right) & =W_{v e_{2}}\left(K_{1}+P_{n}\right)=4 n^{2}-5 n+3 .
\end{aligned}
$$

Corollary 3.4. For $n \geq 4$,

$$
\begin{aligned}
W_{v e_{1}}\left(W_{n+1} ; q\right) & =W_{v e_{1}}\left(K_{1}+C_{n}, q\right)=4 n+n(n+2) q+n(n-4) q^{2}, \\
W_{v e_{2}}\left(W_{n+1} ; q\right) & =W_{v e_{2}}\left(K_{1}+C_{n}, q\right)=7 n q+n(2 n-5) q^{2}, \\
W_{v e_{1}}\left(W_{n+1}\right) & =W_{v e_{1}}\left(K_{1}+C_{n}\right)=3 n(n-2), \\
W_{v e_{2}}\left(W_{n+1}\right) & =W_{v e_{2}}\left(K_{1}+C_{n}\right)=n(4 n-3) .
\end{aligned}
$$

The windmill graph $D_{n}^{(m)}$ is the graph obtained by taking $m$ copies of the complete graph $K_{n}$ with a vertex in common. The case $n=3$ therefore corresponds to the Dutch windmill graph. One can easily see that, the windmill graph $D_{n}^{(m)}$ is the suspension of $m$ copies of $K_{n-1}$. So, by Corollary 3.1, we get the following results. 
Corollary 3.5. For $n \geq 3$ and $m \geq 1$,

$$
\begin{aligned}
W_{v e_{1}}\left(D_{n}^{(m)} ; q\right) & =\frac{1}{2} m(n-1)\left[2 n+\left(2 m(n-1)+n^{2}-4 n+2\right) q+(m-1)(n-1)(n-2) q^{2}\right], \\
W_{v e_{2}}\left(D_{n}^{(m)} ; q\right) & =\frac{1}{2} m n(n-1)\left[n q+(n-1)(m-1) q^{2}\right], \\
W_{v e_{1}}\left(D_{n}^{(m)}\right) & =\frac{1}{2} m(n-1)\left[2 m(n-1)^{2}-n(n-2)-2\right], \\
W_{v e_{2}}\left(D_{n}^{(m)}\right) & =\frac{1}{2} m n(n-1)[2 m(n-1)-(n-2)] .
\end{aligned}
$$

Now consider a complete $n$-partite graph $G=K_{m_{1}, m_{2}, \ldots, m_{n}}$ containing $|V(G)|=\sum_{i=1}^{n} m_{i}$ vertices. By definition of this graph, $V(G)$ can be partitioned into subsets $V_{1}, V_{2}, \ldots, V_{n}$ such that for every $i, 1 \leq i \leq n$, there is no edge between the vertices of $V_{i}$. It is easy to see that, $K_{m_{1}, m_{2}, \ldots, m_{n}}=\bar{K}_{m_{1}}+\bar{K}_{m_{2}}+\cdots+\bar{K}_{m_{n}}$. Using Theorem 2.1 and Corollary 2.2, we can obtain the formulas for the vertex-edge Wiener polynomials and indices of $K_{m_{1}, m_{2}, \ldots, m_{n}}$.

Corollary 3.6. Let $G=K_{m_{1}, m_{2}, \ldots, m_{n}}, \mu_{1}=\sum_{i=1}^{n} m_{i}, \mu_{2}=\sum_{i=1}^{n} m_{i}^{2}$, and $\mu_{3}=\sum_{i=1}^{n} m_{i}^{3}$. Then $W_{v e_{1}}(G ; q)=\mu_{1}^{2}-\mu_{2}+\left[\mu_{3}+\mu_{1}^{2}\left(\mu_{1}-1\right)-\mu_{2}\left(2 \mu_{1}-1\right)\right] q$,

$W_{\nu e_{2}}(G ; q)=\left[2 \mu_{3}-\mu_{2}\left(3 \mu_{1}+1\right)+\mu_{1}^{2}\left(\mu_{1}+1\right)\right] q-\left[\mu_{3}-\mu_{2}\left(\mu_{1}+1\right)+\mu_{1}^{2}\right] q^{2}$,

$W_{v e_{1}}(G)=\mu_{3}-\mu_{2}\left(2 \mu_{1}-1\right)+\mu_{1}^{2}\left(\mu_{1}-1\right)$,

$W_{v e_{2}}(G)=\left(\mu_{1}-1\right)\left(\mu_{1}^{2}-\mu_{2}\right)$.

Now, we turn our attention toward coronas. Coronas sometimes appear in chemical literature as plerographs of the usual hydrogen-suppressed molecular graphs known as kenographs. Interesting classes of graphs can be obtained by specializing the components in the corona product. For a given graph $G$, the graph $K_{2} \circ G$ is called the bottleneck graph of $G$. Using Theorem 2.5 and Corollary 2.6, we obtain the following results.

Corollary 3.7. Let $G$ be a graph of order $n$ and size e. Then

$W_{v e_{1}}\left(K_{2} \circ G ; q\right)=2(2 n+2 e+1)+2\left[n(n+1)-e+M_{1}(G)-3 \Delta(G)\right] q+2[n(n+e)+e+3 \Delta(G)-$ $\left.M_{1}(G)\right] q^{2}+2 n e q^{3}$,

$W_{\nu e_{2}}\left(K_{2} \circ G ; q\right)=2[2 n+5 e+3 \Delta(G)+1] q+2[n(n+1)+e(n-3)-3 \Delta(G)] q^{2}+2 n(n+e) q^{3}$,

$W_{\nu e_{1}}\left(K_{2} \circ G\right)=6 n^{2}+2 n+10 n e+2 e+6 \Delta(G)-2 M_{1}(G)$,

$W_{\nu e_{2}}\left(K_{2} \circ G\right)=10 n^{2}+8 n+10 n e-2 e-6 \Delta(G)+2$.

In particular, we can obtain the formulas for the bottleneck graph of $P_{n}$.

Corollary 3.8. For $n \geq 2$,

$$
\begin{aligned}
W_{v e_{1}}\left(K_{2} \circ P_{n} ; q\right) & =2 n(n-1) q^{3}+2\left(2 n^{2}-4 n+5\right) q^{2}+2\left(n^{2}+4 n-5\right) q+2(4 n-1), \\
W_{v e_{2}}\left(K_{2} \circ P_{n} ; q\right) & =2 n(2 n-1) q^{3}+2\left(2 n^{2}-3 n+3\right) q^{2}+2(7 n-4) q, \\
W_{v e_{1}}\left(K_{2} \circ P_{n}\right) & =16 n^{2}-14 n+10, \\
W_{v e_{2}}\left(K_{2} \circ P_{n}\right) & =20 n^{2}-4 n+4 .
\end{aligned}
$$


For a given graph $G$, the graph $G \circ \bar{K}_{t}$ is called the $t$-thorny graph of $G$ [21]. Using Theorem 2.5 and Corollary 2.6, we can obtain the vertex-edge Wiener polynomials and indices for $t$-thorny graphs.

Corollary 3.9. Let $G$ be a graph of order $n$ and size e. Then

$$
\begin{aligned}
W_{v e_{1}}\left(G \circ \bar{K}_{t}, q\right) & =(t q+1) W_{v e_{1}}(G ; q)+2 t(t q+1) W(G ; q)+n t(t-1) q+2 n t, \\
W_{v e_{2}}\left(G \circ \bar{K}_{t} ; q\right) & =(t q+1) W_{v e_{2}}(G ; q)+2 t q(t q+1) W(G ; q)+n t(t-1) q^{2}+2 n t q, \\
W_{v e_{1}}\left(G \circ \bar{K}_{t}\right) & =(t+1) W_{v e_{1}}(G)+2 t(t+1) W(G)+n t(n t+e-1), \\
W_{v e_{2}}\left(G \circ \bar{K}_{t}\right) & =(t+1) W_{v e_{2}}(G)+2 t(t+1) W(G)+n t(2 n t+n+e-1) .
\end{aligned}
$$

We use known results for the vertex Wiener index and polynomial of paths and cycles [24], our results for their vertex-edge Wiener indices and polynomials [1], and Corollary 3.9 to obtain the formulas for the $t$-thorny path $P_{n} \circ \bar{K}_{t}$ and $t$-thorny cycle $C_{n} \circ \bar{K}_{t}$.

Corollary 3.10. For $n \geq 1$,

$$
\begin{aligned}
W_{v e_{1}}\left(P_{n} \circ \bar{K}_{t} ; q\right)= & 2(t+1)^{2} \sum_{k=1}^{n}(n-k) q^{k}+2\left(t^{2}-1\right) \sum_{k=1}^{n} q^{k}+2(n t+n-1)-n t(t+1) q+2 q^{n}, \\
W_{v e_{2}}\left(P_{n} \circ \bar{K}_{t} ; q\right)= & 2(t+1)^{2} \sum_{k=1}^{n}(n-k) q^{k}+4 t(t+1) \sum_{k=1}^{n} q^{k}-2 t(n t+n+t) q-n t(t+1) q^{2} \\
& +2 t^{2} q^{n+1} \\
W_{v e_{1}}\left(P_{n} \circ \bar{K}_{t}\right)= & \frac{n}{3}\left[(t+1)^{2} n^{2}+3 n\left(t^{2}-1\right)-\left(t^{2}+5 t-2\right)\right], \\
W_{v e_{2}}\left(P_{n} \circ \bar{K}_{t}\right)= & \frac{n}{3}\left[(t+1)^{2} n^{2}+6 t(t+1) n-\left(t^{2}+8 t+1\right)\right] .
\end{aligned}
$$

Corollary 3.11. For $n \geq 3$,

$$
\begin{gathered}
W_{v e_{1}}\left(C_{n} \circ \bar{K}_{t} ; q\right)= \begin{cases}2 n(t+1)^{2} \sum_{k=1}^{\frac{n+1}{2}} q^{k}-n q^{\frac{n-1}{2}}[(3 t+2) q+1] & n \text { is odd } \\
-n(t+1)(t q-2), & \text { n is even } \\
2 n(t+1)^{2} \sum_{k=1}^{\frac{n}{2}} q^{k}+n q^{\frac{n}{2}}\left(t^{2} q-t-2\right) & \\
-n(t+1)(t q-2), & n \text { is odd }\end{cases} \\
W_{v e_{2}}\left(C_{n} \circ \bar{K}_{t} ; q\right)= \begin{cases}2 n(t+1)^{2} \sum_{k=1}^{\frac{n+1}{2}} q^{k}+n q^{\frac{n-1}{2}}\left[2 t^{2} q^{2}+(t-2) q+1\right] \\
-n t(t+1) q(q+2), \\
2 n(t+1)^{2} \sum_{k=1}^{\frac{n}{2}} q^{k}+n t q^{\frac{n}{2}+1}(t q+2 t+3) & n \text { is even } \\
-n t(t+1) q(q+1), & n \text { is odd },\end{cases} \\
W_{\nu e_{1}}\left(C_{n} \circ \bar{K}_{t}\right)=\left\{\begin{array}{l}
\frac{n}{4}\left[n^{2}(t+1)^{2}+2 n\left(2 t^{2}+t-1\right)-\left(t^{2}+4 t-1\right)\right] \\
\frac{n}{4}\left[n^{2}(t+1)^{2}+2 n\left(2 t^{2}+t-1\right)-4 t\right]
\end{array}\right. \text { nis even, }
\end{gathered}
$$




$$
W_{v e_{2}}\left(C_{n} \circ \bar{K}_{t}\right)=\left\{\begin{array}{l}
\frac{n}{4}\left[n^{2}(t+1)^{2}+2 n\left(4 t^{2}+5 t+1\right)-\left(t^{2}+8 t+3\right)\right] \quad n i s \text { odd }, \\
\frac{n}{4}\left[n^{2}(t+1)^{2}+2 n\left(4 t^{2}+5 t+1\right)-4 t\right] \quad n \text { is even } .
\end{array}\right.
$$

\section{Conclusions}

In this paper, we study the behavior of vertex-edge Wiener polynomials and indices under the join and corona product of graphs, and compute them for some classes of graphs such as suspensions, bottlenecks, and thorny graphs. Nevertheless, there are still many classes of chemically interesting and relevant graphs not covered by our approach. It would be interesting to find closed formulas for the vertex-edge Wiener indices of various classes of chemical graphs and nanostructures. In order to achieve that goal, further research into mathematical properties of the vertex-edge Wiener polynomials under other graph operations such as the Cartesian product and rooted product will be necessary.

\section{Acknowledgement}

The authors would like to thank the referee for his/her careful reading and useful suggestions which led us to improve the paper. Partial support by the Center of Excellence of Algebraic Hyper-structures and its Applications of Tarbiat Modares University (CEAHA) is gratefully acknowledged by the second author (AI).

\section{References}

[1] M. Azari and A. Iranmanesh, Computation of the edge Wiener indices of the sum of graphs, Ars Combin., 100 (2011), 113-128.

[2] M. Azari and A. Iranmanesh, Computing Wiener-like topological invariants for some composite graphs and some nanotubes and nanotori, In: I. Gutman, (Ed.), Topics in Chemical Graph Theory, Univ. Kragujevac, Kragujevac, 2014, 69-90.

[3] M. Azari and A. Iranmanesh, The second edge-Wiener index of some composite graphs, Miskolc Math. Notes. 15(2) (2014), 305-316.

[4] M. Azari, A. Iranmanesh and A. Tehranian, A method for calculating an edge version of the Wiener number of a graph operation, Util. Math., 87 (2012), 151-164.

[5] M. Azari, A. Iranmanesh and A. Tehranian, Maximum and Minimum polynomials of a composite graph, Austral. J. Basic Appl. Sci., 5 (9) (2011), 825-830.

[6] M. Azari, A. Iranmanesh and A. Tehranian, Two topological indices of three chemical structures, MATCH Commun. Math. Comput. Chem., 69 (2013), 69-86.

[7] P. Dankelmann, I. Gutman, S. Mukwembi and H. C. Swart, The edge Wiener index of a graph, Discrete Math., 309 (2009), 3452-3457.

[8] M. R. Darafsheh and M. H. Khalifeh, Calculation of the Wiener, Szeged, and PI indices of a certain nanostar dendrimer, Ars Combin., 100 (2011), 289-298.

[9] M. V. Diudea, Hosoya polynomial in tori, MATCH Commun. Math. Comput. Chem., 45 (2002), 109-122.

[10] M. V. Diudea, Wiener index of dendrimers, MATCH Commun. Math. Comput. Chem., 32 (1995), 71-83.

[11] T. Došlić, Vertex-Weighted Wiener polynomials for composite graphs, Ars Math. Contemp., 1 (2008), 66-80. 
[12] I. Gutman, A new method for the calculation of the Wiener number of acyclic molecules, J. Mol. Struct. (Theochem). 285 (1993), 137-142.

[13] I. Gutman, Calculating the Wiener number: the Doyle-Graver method, J. Serb. Chem. Soc., 58 (1993), 745-750.

[14] I. Gutman, Hosoya polynomial and the distance of the total graph of a tree, Univ. Beograd. Publ. Elektrotehn. Fak. Ser. Mat., 10 (1999), 53-58.

[15] I. Gutman and O. E. Polansky, Mathematical Concepts in Organic Chemistry, Springer, Berlin, 1986.

[16] I. Gutman and N. Trinajstić, Graph theory and molecular orbitals, Total $\pi$-electron energy of alternant hydrocarbons, Chem. Phys. Lett., 17 (1972), 535-538.

[17] H. Hosoya, On some counting polynomials in Chemistry, Discrete Appl. Math., 19 (1988), 239-257.

[18] A. Iranmanesh and M. Azari, Edge-Wiener descriptors in chemical graph theory: A survey, Curr. Org. Chem., 19 (3) (2015), 219-239.

[19] A. Iranmanesh, I. Gutman, O. Khormali and A. Mahmiani, The edge versions of Wiener index, MATCH Commun. Math. Compute. Chem., 61 (2009), 663-672.

[20] M. H. Khalifeh, H. Yousefi-Azari, A. R. Ashrafi and S. G. Wagner, Some new results on distance-based graph invariants, European J. Combin., 30 (2009), 1149-1163.

[21] D. J. Klein, T. Došlić and D. Bonchev, Vertex-weightings for distance moments and thorny graphs, Discrete Appl. Math., 155 (2007), 2294-2302.

[22] M. J. Nadjafi-Arani, H. Khodashenas and A. R. Ashrafi, Relationship between edge Szeged and edge Wiener indices of graphs, Glas. Mat. Ser. III, 47(67) (2012), 21-29.

[23] R. Nasiri, H. Yousefi-Azari, M. R. Darafsheh and A. R. Ashrafi, Remarks on the Wiener index of unicyclic graphs, J. Appl. Math. Comput., 41 (1-2) (2013), 49-59.

[24] B. E. Sagan, Y. Yeh and P. Zhang, The Wiener polynomial of a graph, Inter. J. Quantum Chem., 60 (1996), 959-969.

[25] N. Trinajstić, Chemical Graph Theory, CRC Press, Boca Raton, FL, 1992.

[26] H. Wiener, Structural determination of paraffin boiling points, J. Amer. Chem. Soc., 69 (1947), 17-20.

[27] H. Wiener, Correlation of heats of isomerization and differences in heats of vaporization of isomers among the paraffin hydrocarbons, J. Amer. Chem. Soc., 69 (1947), 2636-2638.

[28] Z. Yarahmadi, T. Došlić and A. R. Ashrafi, The bipartite edge frustration of composite graphs, Discrete Appl. Math., 158 (2010), 1551-1558.

Department of Mathematics, Kazerun Branch, Islamic Azad University, P. O. Box: 73135-168, Kazerun, Iran.

E-mail: azari@kau.ac.ir

Department of Pure Mathematics, Faculty of Mathematical Sciences, Tarbiat Modares University, P. O. Box: 14115137, Tehran, Iran.

E-mail: iranmanesh@modares.ac.ir 